\title{
Occupational type affects the receipt of breast cancer adjuvant chemotherapy in China
}

\author{
ZHENGZHI ZHU $^{1,2}$, QIANG HUO ${ }^{1}$, SHENGYING WANG ${ }^{3}$ and QIFENG YANG $^{1}$ \\ ${ }^{1}$ Department of Breast Surgery, Qilu Hospital of Shandong University, Jinan, Shandong 250012; \\ ${ }^{2}$ Department of Oncology Surgery, First Affiliated Hospital of Bengbu Medical College, Bengbu, Anhui 233004; \\ ${ }^{3}$ Medical Center of Breast Disease, Anhui Tumor Hospital, Anhui Medical University, Hefei, Anhui 230001, P.R. China
}

Received April 27, 2015; Accepted July 7, 2015

DOI: 10.3892/ol.2015.3571

\begin{abstract}
Adjuvant chemotherapy has been demonstrated to improve the prognosis of patients with early-stage breast cancer; however, the high cost and side effects associated with this treatment may discourage patients from receiving it. The present study assessed the candidate factors that may influence decisions regarding postoperative adjuvant chemotherapy in females with early-stage breast cancer. Patients diagnosed with invasive breast cancer between January 2000 and December 2007 were enrolled in the study. Information about the patients, including socio-demographic factors, clinicopathological characteristics and receipt of adjuvant chemotherapy, was obtained from their medical records. Overall, 434 out of 1,296 (33.5\%) patients with breast cancer decided against receiving adjuvant chemotherapy. Receipt of chemotherapy was significantly associated with the age of the patient at the time of diagnosis $(\mathrm{P}=0.029)$, occupational type $(\mathrm{P}=0.023)$, and lymph node status $(\mathrm{P}<0.001)$. Moderate associations were also observed between receipt of adjuvant chemotherapy and the patient's family history of cancer $(\mathrm{P}=0.055)$ and hormone-receptor status $(\mathrm{P}=0.075)$. The results of the present study suggest that the occupational type of the patient is associated with receipt of adjuvant chemotherapy in China. This observation may provide a novel strategy for physicians to improve patient's compliance regarding adjuvant chemotherapy. Further studies in additional developing countries are required in order to validate these observations.
\end{abstract}

Correspondence to: Professor Qifeng Yang, Department of Breast Surgery, Qilu Hospital of Shandong University, 107 West Wenhua Road, Jinan, Shandong 250012, P.R. China

E-mail: qifengy@gmail.com

Professor Shengying Wang, Medical Center of Breast Disease, Anhui Tumor Hospital, Anhui Medical University, 107 Huan-hu East Road, Hefei, Anhui 230001, P.R. China

E-mail: wangshengying61@163.com

Key words: adjuvant chemotherapy, breast cancer, occupational types, epidemiology, retrospective study

\section{Introduction}

Breast cancer is one of the most frequently diagnosed types of cancer among females worldwide. The incidence and mortality rate of breast cancer are decreasing in certain developed countries, but are rising in the majority of developing regions, such as Asia and Africa $(1,2)$. In recent years, breast cancer has become one of the leading causes of cancer-associated mortality among females in China, and is the most frequently diagnosed type of malignant tumor $(3,4)$. With the development of various breast cancer treatments, conventional strategies, including radiotherapy, surgery and chemotherapy, provide patients with multiple therapeutic options.

Adjuvant chemotherapy is usually administered following surgery, and is considered one of the standard treatments for invasive breast cancer $(5,6)$. Previous studies have suggested that adjuvant chemotherapy may improve the prognosis of patients with postoperative breast cancer (7-9). The use of adjuvant chemotherapy significantly prolongs disease-free and overall survival among patients with breast cancer but also can cause long-term side effects, such as suppression of ovarian function with premature menopause. This may result in loss of childbearing potential, menopause symptoms, such as hot flushes and genitourinary dysfunctions, infertility and prolonged exposure to menopausal risks, including osteoporosis and cardiovascular disease, causing physical as well as psychological distress $(10,11)$. For these reasons, certain patients, particularly young women, choose not to receive chemotherapy. Furthermore, the elevated cost of chemotherapy may discourage patients with breast cancer from accepting this effective treatment, particularly in economically developing countries such as China (12-15). Numerous studies have indicated that factors such as ethnicity and marital status may impact the receipt of adjuvant chemotherapy among patients with breast cancer in economically developed countries $(16,17)$. A previous study reported that age, marital status, occupational type and economic level could significantly influence the receipt of chemotherapy (18). However, Lipscomb et al (16) reported that the ratio of individuals that completed chemotherapy versus those who did not was not significantly affected by socioeconomic $(\mathrm{P}=0.447)$ or rural status $(\mathrm{P}=0.165)$. Howecer, it still remains uncertain 
whether occupational type is be an influencing factor on the receipt of chemotherapy. In addition, similar studies have not been conducted in developing countries thus far. In the present study, possible factors influencing the receipt of adjuvant chemotherapy to target breast cancer in China were explored and evaluated.

\section{Materials and methods}

Patient selection. Females diagnosed with invasive breast cancer between January 2000 and December 2007 were enrolled in the present study. Diagnosis of breast cancer was confirmed by surgery and pathological examinations at Qilu Hospital of Shandong University (Jinan, China). Information about the participants was retrieved from their medical records. Previous studies have suggested that receipt of neoadjuvant chemotherapy may disturb the receipt of breast cancer adjuvant chemotherapy $(14,16,17)$. Therefore, patients who had received neoadjuvant chemotherapy prior to surgery, or those who did not meet the clinical criteria for consideration of adjuvant chemotherapy according to the recommended guidelines, were excluded from the study (5).

Data collection. Socio-demographic factors and clinicopathological characteristics of the candidates, including age at the time of diagnosis, menstrual status, occupational type, comorbidities, history of benign breast disease, family history of cancer, tumor size, lymph node metastasis, hormone receptor status, and tumor stage, were retrieved and coded anonymously for analysis in the present study. Data collection was performed independently by 2 individuals. All of the participants provided their informed consent prior to inclusion in the study. The present study was approved by the ethical committees of the Qilu Hospital of Shandong University and Bengbu Medical College (Bengbu, China).

Variables. Clinicopathological characteristics, such as estrogen (ER) and progesterone receptor (PR) status, were assessed by immunohistochemical staining, and tumors were classified according to the pathological tumor-node-metastasis (pTNM) staging system (18). Comorbidities included cardiovascular disease, tuberculosis, rheumatism and other chronic conditions excluding tumors. Patients without menses for $>6$ months were classified as postmenopausal individuals. The variables were measured at the initial time of treatment.

Immunohistochemical staining. Briefly, the tissue sections $(4-\mu \mathrm{m})$ were deparaffinized and rehydrated, followed by antigen retrieval with pH 6.0 citrate buffer (ZSGB-BIO, Beijing, China). Endogenous peroxidase activity was inhibited with $3 \% \mathrm{H}_{2} \mathrm{O}_{2}$ for $15 \mathrm{~min}$ and the sections were incubated with $10 \%$ normal goat serum (ZSGB-BIO) to block non-specific binding. After incubation with monoclonal rabbit anti-human ER (cat. no. ZA-0102)/PR (cat. no. ZA-0255) antibodies (ZSGB-BIO) at $4^{\circ} \mathrm{C}$ overnight, the sections were washed, treated with biotinylated polyclonal goat anti-rabbit anti-immunoglobulin antibody (cat. no. SP-9001; ZSGB-BIO) for $20 \mathrm{~min}$ and reacted with horseradish peroxidase-conjugated streptavidin. Subsequently, a the liquid DAB
Table I. Characteristics of the patients ${ }^{\mathrm{a}}$.

\begin{tabular}{lc}
\hline Variable & No. cases (\%) \\
\hline Age & \\
Mean \pm SD (years) & $50.01 \pm 11.22$ \\
Ethnicity & \\
Asian & $1,296(100 \%)$ \\
Menopausal status & \\
Premenopausal & $620(47.8 \%)$ \\
Postmenopausal & $676(52.2 \%)$ \\
Occupational types & \\
Peasant & $341(26.3 \%)$ \\
Laborer & $472(36.4 \%)$ \\
Civil servant and cadre & $174(13.4 \%)$ \\
School teacher & $76(5.9 \%)$ \\
Medical staff & $37(2.9 \%)$ \\
Housewife & $126(9.7 \%)$ \\
Other/unknown & $70(5.4 \%)$
\end{tabular}

Comorbid conditions

0

$914(70.5 \%)$

$\geq 1$

$382(29.5 \%)$

History of breast disease

0

$1,178(90.9 \%)$

$\geq 1$

$118(9.1 \%)$

Family history of cancer

0

$1,193(92.1 \%)$

$103(7.9 \%)$

$\geq 1$

$1,084(83.6 \%)$

201 (15.5\%)

737 (56.9\%)

$558(43.1 \%)$

Positive

$187(14.4 \%)$

$660(50.9 \%)$

$\mathrm{ER}^{-}$and $\mathrm{PR}^{-}$

$\mathrm{ER}^{+}$and/or $\mathrm{PR}^{+}$

$171(13.2 \%)$

$927(71.5 \%)$

198 (15.3\%)

III

$434(33.5 \%)$

$862(66.5 \%)$

Receipt of adjuvant chemotherapy

No

Yes

and hormone

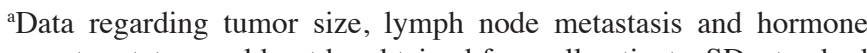
receptor status could not be obtained from all patients. SD, standard deviation; ER, estrogen receptor; PR, progesterone receptor.

substrate/chromogen system (Maixin Bio, Fuzhou, China) was used to stain the sections, prior to counterstaining with hematoxylin (Beijing Solarbio Science \& Technology Co., Ltd., Beijing, China). 
Table II. Univariate analysis of factors associated with the receipt of adjuvant chemotherapy ${ }^{\mathrm{a}}$.

\begin{tabular}{|c|c|c|c|}
\hline Variable & $\begin{array}{l}\text { No. cases } \\
(\mathrm{N}=1,296)\end{array}$ & $\begin{array}{l}\text { Received adjuvant chemotherapy } \\
\qquad(\%, \mathrm{~N}=862)\end{array}$ & $\chi^{2}(\mathrm{P}$-value $)$ \\
\hline \multicolumn{4}{|l|}{ Age (years) } \\
\hline$<50$ & 691 & 68.6 & \multirow[t]{3}{*}{0.018} \\
\hline $50-70$ & 529 & 65.8 & \\
\hline$>70$ & 76 & 52.6 & \\
\hline \multicolumn{4}{|l|}{ Occupational types } \\
\hline Peasant & 341 & 61.9 & \multirow{5}{*}{0.053} \\
\hline Laborer & 472 & 67.6 & \\
\hline Civil servants and cadre & 174 & 64.9 & \\
\hline School teacher and medical staff & 113 & 76.1 & \\
\hline Housewife & 126 & 70.6 & \\
\hline \multicolumn{4}{|l|}{ Menopausal status } \\
\hline Premenopausal & 620 & 67.9 & \multirow[t]{2}{*}{0.310} \\
\hline Postmenopausal & 676 & 65.2 & \\
\hline \multicolumn{4}{|l|}{ Comorbidities } \\
\hline 0 & 914 & 66.7 & \multirow[t]{2}{*}{0.789} \\
\hline$\geq 1$ & 382 & 66.0 & \\
\hline \multicolumn{4}{|l|}{ History of breast disease } \\
\hline No & 1178 & 66.7 & \multirow[t]{2}{*}{0.611} \\
\hline Yes & 118 & 64.4 & \\
\hline \multicolumn{4}{|l|}{ Family history of cancer } \\
\hline No & 1193 & 65.6 & \multirow[t]{2}{*}{0.022} \\
\hline Yes & 103 & 76.7 & \\
\hline \multicolumn{4}{|l|}{ Tumor size $(\mathrm{cm})$} \\
\hline$\leq 2$ & 1084 & 67.2 & \multirow[t]{2}{*}{0.337} \\
\hline$>2$ & 201 & 63.7 & \\
\hline \multicolumn{4}{|l|}{ Lymph node metastasis } \\
\hline Negative & 737 & 62.4 & \multirow[t]{2}{*}{$<0.001$} \\
\hline Positive & 558 & 72.0 & \\
\hline \multicolumn{4}{|l|}{ Tumor stage } \\
\hline I & 171 & 63.7 & \multirow[t]{3}{*}{0.396} \\
\hline II & 927 & 67.6 & \\
\hline III & 198 & 63.6 & \\
\hline \multicolumn{4}{|l|}{ Hormone receptor status } \\
\hline $\mathrm{ER}^{-}$and $\mathrm{PR}^{-}$ & 187 & 61.5 & \multirow[t]{2}{*}{0.095} \\
\hline $\mathrm{ER}^{+}$and/or $\mathrm{PR}^{+}$ & 660 & 68.0 & \\
\hline
\end{tabular}

${ }^{a}$ Data regarding tumor size, lymph node metastasis and hormone receptor status could not be obtained from all patients. ER, estrogen receptor; PR, progesterone receptor.

Statistical analysis. Univariate analyses were conducted for each of the variables and the receipt of adjuvant chemotherapy using $\chi^{2}$ tests, accompanied by 2 -sided P-values. Multivariate logistic regression analyses, performed using the Hosmer-Lemeshow model, evaluated the associations between these parameters, which were expressed as adjusted odds ratios (ORs) with $95 \%$ confidence intervals (CIs). $\mathrm{P}>0.5$ was considered as high goodness-of-fit. Statistical analyses were performed using the IBM SPSS Statistics software, version 20.0.0 (IBM SPSS, Armonk, NY, USA), and based on 2-tailed probability. $\mathrm{P}<0.05$ was considered to indicate a statistically significant difference.

\section{Results}

A total of 1,296 females diagnosed with invasive breast cancer (pTNM stage I-III) were enrolled in the present study (Table I). All of the patients were of Asian descent, and their age ranged from 23 to 85 years. Among the participants, 434 (33.5\%) decided against receiving adjuvant chemotherapy. 
Table III. Multivariate analysis of candidate factors associated with the receipt of adjuvant chemotherapy.

\begin{tabular}{|c|c|c|c|}
\hline Variable & $\mathrm{OR}^{\mathrm{a}}$ & $95 \% \mathrm{CI}^{\mathrm{a}}$ & Adjusted P-value \\
\hline Age (years) & 0.988 & $0.978-0.999$ & 0.029 \\
\hline Occupational types & & & 0.023 \\
\hline Peasant & 1.000 & & \\
\hline Laborer & 1.377 & $1.022-1.857$ & 0.036 \\
\hline Civil servants and cadre & 1.273 & $0.862-1.881$ & 0.226 \\
\hline School teacher and medical staff & 2.215 & $1.349-3.635$ & 0.002 \\
\hline Housewife & 1.669 & $1.061-2.627$ & 0.027 \\
\hline \multicolumn{4}{|l|}{ Family history of cancer } \\
\hline No & 1.000 & & \\
\hline Yes & 1.598 & $0.990-2.578$ & 0.055 \\
\hline \multicolumn{4}{|l|}{ Lymph node metastasis } \\
\hline Negative & 1.000 & & \\
\hline Positive & 1.633 & $1.276-2.089$ & $<0.001$ \\
\hline \multicolumn{4}{|l|}{ Hormone receptor status } \\
\hline $\mathrm{ER}^{-}$and $\mathrm{PR}^{-}$ & 1.000 & & \\
\hline $\mathrm{ER}^{+}$and/or $\mathrm{PR}^{+}$ & 1.366 & $0.970-1.925$ & 0.075 \\
\hline
\end{tabular}

${ }^{a}$ Adjusted using multivariate logistic regression model. OR, odds ratios; CI, confidence interval; ER, estrogen receptor, PR, progesterone receptor.

In order to assess the factors influencing the receipt of breast cancer adjuvant chemotherapy, all the possible factors were included in the univariate analyses. As indicated in Table II, certain factors, including the patient's menopausal status $(\mathrm{P}=0.310)$, comorbidities $(\mathrm{P}=0.789)$, history of breast disease $(\mathrm{P}=0.611)$, tumor size $(\mathrm{P}=0.337)$ or tumor stage $(\mathrm{P}=0.396)$, were not significantly associated with the receipt of adjuvant chemotherapy $(P>0.1)$. Therefore, these parameters were temporarily excluded from the multivariate analysis.

Adjusted values were estimated by controlling covariates in a multivariate logistic regression model, including the patient's age at the time of diagnosis, occupational type, family history of cancer, lymph node metastasis and hormone receptor status. The results suggest that the patient's age at the time of diagnosis $(\mathrm{OR}=0.988,95 \% \mathrm{CI}=0.978-0.999$, adjusted $\mathrm{P}=0.029)$, occupational type (adjusted $\mathrm{P}=0.023$ ) and lymph node status $(\mathrm{OR}=1.633,95 \% \mathrm{CI}=1.276-2.089$, adjusted $\mathrm{P}<0.001)$ were statistically associated with the receipt of adjuvant chemotherapy $(\mathrm{P}<0.05$; Table III). Covariates, including patient's family history of cancer and hormone-receptor status were observed to be moderately associated with the receipt of adjuvant chemotherapy $(0.05<\mathrm{P}<0.10)$. Subsequently, the multivariate models were re-estimated using all of the variables, including patient's age, occupational type, menopausal status, comorbidities, history of benign breast disease, family history of cancer, tumor size, lymph node status, tumor stage and hormone receptor status. The results suggest that the statistical pattern was not altered (data not shown).

The logistic regression models adopted in the present study exhibited high goodness-of-fit (Hosmer-Lemeshow, $\mathrm{P}>0.5)$.

\section{Discussion}

Adjuvant chemotherapy is vital for patients with invasive breast cancer, and has been associated with improved prognosis, as demonstrated by previous experimental data and clinical trials (19-21). Furthermore, consensus guidelines have identified subsets of female patients with invasive breast cancer that benefit from appropriate adjuvant chemotherapy $(5,22,23)$. Previous studies have suggested that numerous factors, including age, comorbidities, and variability in provider recommendation, were associated with the receipt of adjuvant chemotherapy by females in Western countries (24-28). Since adjuvant chemotherapy is generally recommended to patients with invasive breast cancer, cases of carcinoma in situ were not included in the present study. Records of those patients who were recommended chemotherapy but refused it were used to identify and recruit suitable candidates for the present study.

In the present study it was observed that a large number of peasants decided against receiving adjuvant chemotherapy, as compared with laborers, housewives, and, particularly, teachers and health professionals. This may be due to rural-urban disparities in education levels, lifestyles and cultural values of females in China $(15,29,30)$. In economically developing countries such as China, urban females are usually exposed to cultural globalization and better education, as compared with those in rural areas. Therefore, urban females tend to believe in modern medicine and are more familiar with the benefits of adjuvant chemotherapy $(14,15)$. The present study also demonstrated that the patient's age at the time of diagnosis was associated with the rate of receipt of adjuvant chemotherapy (8,31-33). The results suggested that a reduced number of elderly females accepted adjuvant chemotherapy, as compared with young females $(\mathrm{OR}=0.988 /$ year $)$. In practice, 
the tolerance of elderly patients to chemotherapy is reduced, as compared with in young females, which may explain the apparently stronger will for the elderly to adopt a more conservative type of treatment $(22,34,35)$. The fact that a large number of patients with positive lymph node metastasis were willing to receive adjuvant chemotherapy may be due to the importance of lymph node status on patient survival. Consequently, doctors may have encouraged these patients to receive the treatment $(5,6,22)$.

The present study possesses several limitations. Patients who did not receive adjuvant chemotherapy were regarded as incompliant patients, because they were recommended to receive the treatment but refused to do so. However, in certain cases, doctors did not offer chemotherapy to the patients, despite being indicated in the recommended guidelines. In those cases, the patients should not be considered incompliant, despite not receiving adjuvant chemotherapy. The patients who were not recommended to receive chemotherapy by their doctors due to their old age or other reasons, were excluded from the present study. In addition, the level of education of the patients was not included as a confounding variable in the analyses, since the patient registration system and medical records did not contain information regarding the patients' education, and the majority of patients would not provide their certificates of education, since it was considered troublesome, private and usually unnecessary. However, the education levels of the patients may be a relevant factor in the present study.

In conclusion, the results of the present study suggest that the occupational type of patients is independently associated with the receipt of adjuvant chemotherapy in China. This observation may provide a novel strategy for physicians to improve patient's compliance with adjuvant chemotherapy. Further studies in other developing countries are required in order to validate these observations.

\section{Acknowledgements}

The present study was supported by the National Natural Science Foundation of China (grant nos. 30772133, 81072150, 81172529 and 81272903), and the Shandong Science and Technology Development Plan (grant no. 2012GZC22115). The funding bodies did not participate in the study design, data collection and analysis, decision to publish or preparation of the manuscript.

\section{References}

1. Jemal A, Bray F, Center MM, Ferlay J, Ward E and Forman D: Global cancer statistics. CA Cancer J Clin 61: 69-90, 2011.

2. Siegel R, Ma J, Zou Z and Jemal A: Cancer statistics, 2014. CA Cancer J Clin 64: 9-29, 2014.

3. Ziegler RG, Anderson WF and Gail MH: Increasing breast cancer incidence in China: The numbers add up. J Natl Cancer Inst 100: 1339-1341, 2008.

4. Chen W, Zheng R, Zhang S, Zhao P, Li G, Wu L and He J: Report of incidence and mortality in China cancer registries, 2009. Chin J Cancer Res 25: 10-21, 2013.

5. No authors listed: Adjuvant therapy for breast cancer. NIH Consens Statement 17: 1-35, 2000.

6. No authors listed: NIH consensus conference. Treatment of early-stage breast cancer. JAMA 265: 391-395, 1991.

7. Bonadonna $\mathrm{G}$ and Valagussa P: Dose-response effect of adjuvant chemotherapy in breast cancer. N Engl J Med 304: 10-15, 1981.
8. Muss HB, Berry DA, Cirrincione CT, Theodoulou M, Mauer AM, Kornblith AB, Partridge AH, Dressler LG, Cohen HJ, Becker HP, et al; CALGB Investigators: Adjuvant chemotherapy in older women with early-stage breast cancer. N Engl J Med 360: 2055-2065, 2009.

9. Doll R: Strategy for detection of cancer hazards to man. Nature 265: 589-596, 1977.

10. Vanhuyse M,FournierCand Bonneterre J: Chemotherapy-induced amenorrhea: influence on disease-free survival and overall survival in receptor-positive premenopausal early breast cancer patients. Ann Oncol 16: 1283-1288, 2005.

11. Ben-Aharon I, Meizner I, Granot T, Uri S, Hasky N, Rizel S, YerushalmiR,Sulkes A and Stemmer SM: Chemotherapy-induced ovarian failure as a prototype for acute vascular toxicity. Oncologist 17: 1386-1393, 2012.

12. Reinisch M, von Minckwitz G, Harbeck N, Janni W, Kümmel S, Kaufmann M, Elling D, Nekljudova V and Loibl S: Side effects of standard adjuvant and neoadjuvant chemotherapy regimens according to age groups in primary breast cancer. Breast Care (Basel) 8: 60-66, 2013.

13. Rosendahl M, Ahlgren J, Andersen J, Bergh J, Blomquist C, Lidbrink E, Lindman $\mathrm{H}$, Mouridsen $\mathrm{H}$, Bjerre $\mathrm{K}$ and Andersson M: The risk of amenorrhoea after adjuvant chemotherapy for early stage breast cancer is related to inter-individual variations in chemotherapy-induced leukocyte nadir in young patients: Data from the randomised SBG 2000-1 study. Eur J Cancer 45: 3198-3204, 2009.

14. Jingang A: Which future for doctors in China? Lancet 382: 936-937, 2013.

15. Goss PE, Strasser-Weippl K, Lee-Bychkovsky BL, et al: Challenges to effective cancer control in China, India, and Russia. Lancet Oncol 15: 489-538, 2014.

16. Lipscomb J, Gillespie TW, Goodman M, Richardson LC, Pollack LA, Ryerson AB and Ward KC: Black-white differences in receipt and completion of adjuvant chemotherapy among breast cancer patients in a rural region of the US. Breast Cancer Res Treat 133: 285-296, 2012.

17. Hershman DL, Unger JM, Barlow WE, Hutchins LF, Martino S, Osborne CK, Livingston RB and Albain KS: Treatment quality and outcomes of African American versus white breast cancer patients: Retrospective analysis of Southwest Oncology studies S8814/S8897. J Clin Oncol 27: 2157-2162, 2009.

18. National Comprehensive Cancer Network (NCCN): NCCN Clinical Practice Guidelines in Oncology. Breast Cancer. Fort Washington, PA, pp66-69, 2012.

19. Kilickap S and Arslan C: Adjuvant chemotherapy and prognosis in patients with breast cancer. Ann Oncolo 20: 192-193; author reply 193-194, 2009.

20. Fisher ER, Redmond $C$ and Fisher B: Pathologic findings from the National Surgical Adjuvant Breast Project. VIII. Relationship of chemotherapeutic responsiveness to tumor differentiation. Cancer 51: 181-191, 1983.

21. Fisher B and Redmond C: Systemic therapy in node-negative patients: updated findings from NSABP clinical trials. National Surgical Adjuvant Breast and Bowel Project. J Ntl Cancer Inst Monogr: 105-116, 1992.

22. Goldhirsch A, Glick JH, Gelber RD, Coates AS and Senn HJ: Meeting highlights: International Consensus Panel on the Treatment of Primary Breast Cancer. Seventh International Conference on Adjuvant Therapy of Primary Breast Cancer. J Clin Oncol 19: 3817-3827, 2001.

23. Rao RD and Cobleigh MA: Adjuvant endocrine therapy for breast cancer. Oncology (Williston Park) 26: 541-547, 550, 552 passim, 2012.

24. Richardson LC, Tian L, Voti L, Hartzema AG, Reis I, Fleming LE and Mackinnon J: The roles of teaching hospitals, insurance status, and race/ethnicity in receipt of adjuvant therapy for regional-stage breast cancer in Florida. Am J Public Health 96: 160-166, 2006.

25. Silliman RA, Guadagnoli E, Weitberg AB and Mor V: Age as a predictor of diagnostic and initial treatment intensity in newly diagnosed breast cancer patients. J Gerontol 44: M46-M50, 1989.

26. Du XL, Key CR, Osborne C, Mahnken JD and Goodwin JS: Discrepancy between consensus recommendations and actual community use of adjuvant chemotherapy in women with breast cancer. Ann Intern Med 138: 90-97, 2003.

27. Bickell NA, Aufses AH Jr and Chassin MR: The quality of early-stage breast cancer care. Ann Surg 232: 220-224, 2000.

28. Bradley CJ, Given CW and Roberts C: Race, socioeconomic status, and breast cancer treatment and survival. J Natl Cancer Inst 94: 490-496, 2002. 
29. Zhang Y, Gao H, Bu Y, Fan X and Jia J: Factors associated with receipt of adjuvant chemotherapy among married women with breast cancer. World J Surg Oncol 11: 286, 2013.

30. Zhang $\mathrm{Y}, \mathrm{Bu} \mathrm{Y}$ and $\mathrm{Gao} \mathrm{H}$ : Rural-urban disparities of breast cancer patients in China. Med Oncol 30: 387, 2013.

31. Giordano SH, Duan Z, Kuo YF, Hortobagyi GN and Goodwin JS: Use and outcomes of adjuvant chemotherapy in older women with breast cancer. J Clin Oncol 24: 2750-2756, 2006.

32. Buist DS, Chubak J, Prout M, Yood MU, Bosco JL, Thwin SS, Gold HT, Owusu C, Field TS, Quinn VP, et al: Referral, receipt, and completion of chemotherapy in patients with early-stage breast cancer older than 65 years and at high risk of breast cancer recurrence. J Clin Oncol 27: 4508-4514, 2009.
33. Bouchardy C, Rapiti E, Fioretta G, Laissue P, Neyroud-Caspar I, Schäfer P, Kurtz J, Sappino AP and Vlastos G: Undertreatment strongly decreases prognosis of breast cancer in elderly women. J Clin Oncol 21: 3580-3587, 2003.

34. Du X and Goodwin JS: Patterns of use of chemotherapy for breast cancer in older women: Findings from Medicare claims data. J Clin Oncol 19: 1455-1461, 2001.

35. Mandelblatt JS, Sheppard VB, Hurria A, Kimmick G, Isaacs C, Taylor KL, Kornblith AB, Noone AM, Luta G, Tallarico M, et al; Cancer Leukemia Group B: Breast cancer adjuvant chemotherapy decisions in older women: The role of patient preference and interactions with physicians. J Clin Oncol 28: 3146-3153, 2010. 